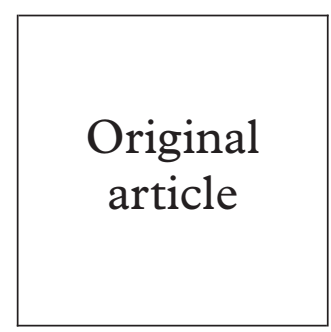

\title{
Seroprevalence and incidence of genital ulcer infections in a rural Ugandan population
}

\author{
A Kamali, A J Nunn, D w Mulder, E Van Dyck, J G Dobbins, J A G Whitworth
}

\section{Medical Research Council Programme on AIDS in \\ Uganda/Uganda Virus \\ Research Institute \\ A Kamali \\ J A G Whitworth}

\section{University College}

London Medical

School, Mortimer

Market Centre,

London

A J Nunn

Department of Social Medicine, Academic

Medical Centre,

University of

Amsterdam,

Netherlands

D W Mulder

Department of

Microbiology, Prince

Leopold Institute of

Tropical Medicine,

Antwerp, Belgium

E Van Dyck

Viral Immunology

Section, CDC, Atlanta,

Georgia, USA

J G Dobbins

Correspondence to:

Dr A Kamali, Medical

Research Council

Programme on AIDS in

Uganda, PO Box 49,

Entebbe, Uganda.

Accepted for publication

18 January 1999

Objectives: To determine age-sex specific seroprevalence and incidence rates of Treponema pallidum, Haemophilus ducreyi, and HSV-2; to assess the association between HIV-1 status and incidence of these STIs; and HSV-2 serostatus with number of lifetime sexual partners.

Methods: Antibodies against HIV-1, T pallidum, $H$ ducreyi, and HSV-2 infections were tested using approximately 1000 paired ( 2 year interval) sera collected from a rural adult (15-54 years) population cohort in south west Uganda.

Results: Overall HIV-1 prevalence was $4.9 \%$. Prevalence for T pallidum was $12.9 \%$ among males and $12.6 \%$ among females. The corresponding rates for $H$ ducreyi were $9.8 \%$ and $7.3 \%$ respectively. HSV-2 prevalence rates were considerably lower in males $(36.0 \%)$ than in females $(71.5 \%)$, $\mathrm{p}<0.001$. Incidence rates for $T$ pallidum per 1000 person years of observation were 8.4 for males and 12.3 for females. The corresponding rates for $H$ ducreyi were 24.6 and 20.0 and for HSV-2 were 73.2 and 122.9 per 1000 person years of observation, respectively. The RR of HSV-2 incidence was 3.69 in HIV seropositive cases versus HIV seronegative after adjusting for age and sex. The corresponding RR for $H$ ducreyi was 3.50 among female HIV positive cases versus negatives with no effect seen in males. Association between HIV-1 prevalence and prevalence of other STIs was significant (Mantel-Haenszel test) for H ducreyi $(\mathrm{p}=0.01)$ and for HSV-2 ( $\mathrm{p}=0.004)$ but not for T pallidum ( $\mathrm{p}>0.4$ ). HSV-2 prevalence was associated with number of lifetime sexual partners (females, $\mathrm{p}=0.003$; males, $\mathrm{p}=0.08$ ).

Conclusions: The results have provided a reliable estimate of the magnitude of the STI problem and demonstrated an association between HIV-1 status and serology of other STIs in a general rural population in sub-Saharan Africa. The study has also highlighted a correlation between HSV-2 seropositivity and number of reported lifetime sexual partners.

(Sex Transm Inf 1999;75:98-102)

Keywords: genital ulcer infections; HIV-1; rural population

\section{Introduction}

Although it is generally recognised that sexually transmitted infections (STIs) contribute a major public health problem, little information exists on the burden of STIs in general populations of sub-Saharan countries. ${ }^{12}$ Studies done on STIs have been mainly on selected populations such as females attending gynaecological, obstetric, and family planning clinics, STI clinic attenders, and among female sex workers. ${ }^{3-5}$ Findings from such studies cannot be generalised to general populations in whom most STIs are either unrecognised or asymptomatic. The scarcity of accurate data is consequently a handicap in designing appropriate control measures. Yet such measures are particularly important in the context of the HIV epidemic since it is now well established that STIs, particularly those causing genital ulceration, facilitate HIV transmission and HIV infection may simultaneously prolong or augment the infectiousness of individuals with STIs. ${ }^{6-9}$ A more meaningful source of data would be from large population serological surveys using the current assays now available for most STIs. Such population surveys done on longitudinal serological studies would also provide data on recent exposures and be useful for surveillance and evaluation of interventions.

In 1994 we published results from an exploratory STD serological study of the adult population of two villages in south west Uganda. ${ }^{10}$ Paired blood samples with an interval of one year from a total of 294 adults were tested. The results indicated that the STIs studied-Treponema pallidum, Haemophilus ducreyi, Chlamydia trachomatis, and herpes simplex virus types 1 and 2-were common. Because of the small numbers the precision of the study was low, particularly for age and sex subgroups. To contribute further to the understanding of the descriptive epidemiology of STIs in rural sub-Saharan populations, we conducted a larger study from adult members of a general population cohort. The specific objectives were to determine the age and sex specific prevalence and incidence rates of serological reactivity to $T$ pallidum, $H$ ducreyi, and herpes simplex virus type 2 (HSV-2); to assess the association between HIV-1 status and incidence of other STIs; and to assess HSV-2 serostatus with number of lifetime sexual partners, as it has been suggested that HSV-2 serology could be a useful indicator in monitoring sexual behaviour changes. ${ }^{10}$

\section{Methods}

In 1989 the Medical Research Council Programme on AIDS in Uganda enrolled a rural population cohort from 15 neighbouring villages in south west Uganda to study the population dynamics of HIV-1 transmission and associated risk factors. The cohort has been 
Table 1 Age-sex seroprevalence* and incidence of T pallidum

\begin{tabular}{|c|c|c|c|c|c|c|}
\hline \multirow[b]{2}{*}{ Age (years) } & \multicolumn{3}{|c|}{ Prevalence } & \multicolumn{3}{|c|}{ Incidence } \\
\hline & $N$ & Pos & $\%$ & Pyot & $I n c$ & Rate \\
\hline \multicolumn{7}{|l|}{ Males: } \\
\hline $15-19$ & 101 & 1 & 1.0 & 183.4 & 0 & 0.0 \\
\hline $20-24$ & 50 & 6 & 12.0 & 128.8 & 1 & 7.8 \\
\hline $25-34$ & 88 & 13 & 14.8 & 165.1 & 1 & 6.1 \\
\hline $35-44$ & 64 & 18 & 28.1 & 120.1 & 1 & 8.3 \\
\hline $45+$ & 54 & 8 & 14.9 & 114.9 & 3 & 26.1 \\
\hline $\begin{array}{l}\text { Total } \\
95 \% \text { CI }\end{array}$ & 357 & 46 & $\begin{array}{l}12.9 \\
(9.6-16.8)\end{array}$ & 712.3 & 6 & $\begin{array}{l}8.4 \\
(3.8-18.7)\end{array}$ \\
\hline \multicolumn{7}{|l|}{ Females: } \\
\hline $15-19$ & 96 & 3 & 3.1 & 181.8 & 1 & 5.5 \\
\hline $20-24$ & 85 & 9 & 10.6 & 181.3 & 2 & 11.0 \\
\hline $25-34$ & 154 & 27 & 17.5 & 333.5 & 3 & 9.0 \\
\hline $35-44$ & 121 & 18 & 14.9 & 234.2 & 4 & 17.1 \\
\hline $45+$ & 82 & 11 & 13.4 & 211.0 & 4 & 19.0 \\
\hline Total & 538 & 68 & 12.6 & 1141.8 & 14 & 12.3 \\
\hline $95 \%$ CI & & & $(10.0-15.7)$ & & & $(7.3-20.7)$ \\
\hline
\end{tabular}

${ }^{\star}$ Both RPR and TPHA positive.

†Person years of observation.

Seroincidence rates per 1000 person years of observation.

followed annually through annual demographic and serological surveys. ${ }^{11}$ The sampling and follow up rate have been described elsewhere ${ }^{12}$ but, briefly, all resident adults (about 5000) in the study area were selected for the main study. Compliance rates were approximately $80 \%$ at baseline survey, $66 \%$ and $60 \%$ for 1990-1 and 1992-3 annual survey rounds respectively. Non-compliance was not, however, cumulative and $88 \%$ of the last round resident population had complied at some time. The present study utilised sera from the main surveys with the objective of selecting and testing a random sample of 1000 adults aged 15-54 years with paired sera from 1990-1 and 1992-3 surveys. Sufficient stored sera were available for testing at least one sample from 924 adults.

In addition to the selected paired samples from the whole population we also tested paired samples from HIV incident subjects at two different time points. In order to increase the number of available subjects we included pre- and post seroconversion samples for the incident cases identified during annual surveys between 1990 and 1994. Sufficient serum samples were available for 42 incident cases.

Testing for HIV-1 antibody was carried out at the Uganda Virus Research Institute, Entebbe, Uganda, using two independent ELISA systems (Recombigen HIV-1 EIA, Cambridge Bioscience, USA, and Wellcozyme HIV-1 Recombinant, Wellcome Diagnostics, UK) and confirmed with western blot using Novopath HIV Immunoblot (Bio-Rad Laboratories, USA) if the ELISA results were discordant or weakly concordant. The quality control procedures and test algorithm are described in detail elsewhere. ${ }^{13}{ }^{14}$ Samples were tested for $T$ pallidum and $H$ ducreyi at the Department of Microbiology, Institute of Tropical Medicine, Antwerp, Belgium. For syphilis serology a rapid plasma reagin card test (Macro-Vue RPR Card Test, BectonDickinson, USA) and a $T$ pallidum microhaemagglutination assay (TPHA, Fujirebio Inc, Japan) were used. Both tests were performed qualitatively and repeated in a quantitative assay if the qualitative test showed reactivity. Serum immunoglobulin G antibodies to $H$ ducreyi were assayed in an ELISA system using an ultrasonicated whole cell antigen. ${ }^{15}$ HSV-2 samples were tested at the Viral Immunology Section, Centers for Disease Control and Prevention, Atlanta, USA. The reactivity against HSV-2 type specific glycoproteins was assessed by western blot technique. ${ }^{16}$ All laboratories received samples blind to subject characteristics. In interpreting reactivity, we followed the manufactures' instructions or, for non-commercial assays, the criteria provided by the laboratory performing the test.

The prevalence estimates have been obtained from serological findings of the first blood sample while incidence rates are based on seroconversions for the specified STIs that occurred in those seronegative at the first assessment. Estimates of incidence rates were obtained using the person years between the first and second serum sample; Poisson regression analyses were used to correct for age and sex differences. Seroincidence was assumed to have occurred midway between the dates of the seronegative and the seropositive samples.

Reported number of life sexual partners from the ongoing medical questionnaire based interviews were obtained only during the 1992-3 annual survey and the data have been linked to HSV-2 serostatus at the time of the second assessment.

\section{Results}

The HIV-1 seroprevalence in the population studied was $4.9 \%$ (95\% CI 3.6-6.5\%), 45 of 924 people assessed.

\section{$T$ PALLIDUM PREVALENCE}

The age-sex prevalence (both RPR and TPHA positive) rates are shown in table 1 . Results for both RPR and TPHA were available for a total of 895 people ( 357 men, 538 women). The overall rates for males $(95 \% \mathrm{CI})$ were $12.9 \%$ (9.6-16.8) and for females $12.6 \%$ (10.0-15.7). The rates increased steeply between the 15-19 and $20-24$ age groups from $1 \%$ to $12 \%$ for males and from $3 \%$ to $11 \%$ for females. The overall increase with age was significant $\left(\chi_{\text {trend }}^{2}\right.$ $=16.53, \mathrm{p}<0.001$ for males, $\chi_{\text {trend }}^{2}=5.67$, $\mathrm{p}=0.02$ for females). After adjusting for age there was no difference in prevalence rates between males and females.

\section{T PALLIDUM INCIDENCE}

Of 735 people initially negative for active syphilis (RPR and/or TPHA negative), and with a serum sample available, 20 (six men, 14 women) seroconverted for both RPR/TPHA. Incidence rates were 8.4 (95\% CI 3.8-18.7) for males and for females 12.3 (95\% CI 7.3-20.7) per 1000 person years of observation. The rates were higher in females than in males for all ages except in those aged 45 or more. The female:male rate ratio was not significant (1.32, 95\% CI 0.51-3.45).

\section{H DUCREYI PREVALENCE}

A total of 893 samples had results for $H$ ducreyi serology. Seroprevalence in males was $9.8 \%$ 
Table 2 Age-sex seroprevalence and incidence of $H$ ducreyi

\begin{tabular}{|c|c|c|c|c|c|c|}
\hline \multirow[b]{2}{*}{ Age (years) } & \multicolumn{3}{|c|}{ Prevalence } & \multicolumn{3}{|c|}{ Incidence } \\
\hline & $N$ & Pos & $\%$ & Pyo* & $I n c$ & Rate \\
\hline \multicolumn{7}{|l|}{ Males: } \\
\hline $15-19$ & 101 & 5 & 5.0 & 172.4 & 4 & 23.2 \\
\hline $20-24$ & 50 & 3 & 6.0 & 129.7 & 1 & 7.7 \\
\hline $25-34$ & 87 & 11 & 12.6 & 170.5 & 6 & 35.2 \\
\hline $35-44$ & 64 & 7 & 10.9 & 143.7 & 1 & 7.0 \\
\hline $45+$ & 54 & 9 & 16.7 & 114.0 & 6 & 52.6 \\
\hline Total & 356 & 35 & 9.8 & 730.3 & 18 & 24.6 \\
\hline $\begin{array}{l}95 \% \text { CI } \\
\text { Females: }\end{array}$ & & & $(6.9-13.4)$ & & & $(11.3-28.6)$ \\
\hline $15-19$ & 96 & 2 & 2.1 & 179.6 & 2 & 6.7 \\
\hline $20-24$ & 85 & 1 & 1.2 & 201.2 & 4 & 19.9 \\
\hline $25-34$ & 153 & 9 & 5.9 & 354.0 & 9 & 25.4 \\
\hline $35-44$ & 121 & 14 & 11.6 & 250.4 & 4 & 16.0 \\
\hline $45+$ & 82 & 13 & 15.9 & 211.9 & 5 & 23.6 \\
\hline Total & 537 & 39 & 7.3 & 1197.1 & 24 & 20.0 \\
\hline $95 \%$ CI & & & $(5.2-9.8)$ & & & (13.4-29.8) \\
\hline
\end{tabular}

$\star$ Person years of observation.

Table 3 Age-sex seroprevalence and incidence of $\mathrm{HSV}-2$

\begin{tabular}{|c|c|c|c|c|c|c|}
\hline \multirow[b]{2}{*}{ Age (years) } & \multicolumn{3}{|c|}{ Prevalence } & \multicolumn{3}{|c|}{ Incidence } \\
\hline & $N$ & Pos & $\%$ & $\mathrm{Pyo}^{\star}$ & $N$ & Rate \\
\hline \multicolumn{7}{|l|}{ Males: } \\
\hline $15-19$ & 101 & 10 & 9.9 & 163.0 & 5 & 30.6 \\
\hline $20-24$ & 52 & 14 & 26.9 & 115.8 & 12 & 103.6 \\
\hline $25-34$ & 89 & 42 & 47.2 & 105.1 & 8 & 76.1 \\
\hline $35-44$ & 66 & 32 & 48.5 & 68.7 & 6 & 87.3 \\
\hline $45+$ & 59 & 34 & 57.6 & 66.4 & 7 & 105.4 \\
\hline Total & 367 & 132 & 36.0 & 519.1 & 38 & 73.2 \\
\hline $95 \% \mathrm{CI}$ & & & $(31.2-41.1)$ & & & (53.4-100.3) \\
\hline \multicolumn{7}{|l|}{ Females: } \\
\hline $15-19$ & 96 & 34 & 35.4 & 116.3 & 11 & 94.6 \\
\hline $20-24$ & 88 & 65 & 73.9 & 55.2 & 12 & 217.4 \\
\hline $25-34$ & 155 & 120 & 77.4 & 75.2 & 7 & 93.1 \\
\hline $35-44$ & 121 & 106 & 87.6 & 33.3 & 3 & 90.1 \\
\hline $45+$ & 81 & 62 & 76.5 & 45.5 & 7 & 153.8 \\
\hline Total & 541 & 387 & 71.5 & 325.5 & 40 & 122.9 \\
\hline $95 \% \mathrm{CI}$ & & & $(67.5-75.3)$ & & & $(90.4-167.1)$ \\
\hline
\end{tabular}

$\star$ Person years of observation.

(6.9-13.4) and in females, $7.3 \%$ (5.2-9.8). The rates were higher in males than females across all age groups except in those aged 35-44 years (table 2). There was a highly significant increase in prevalence by age group in both sexes; in males, $\chi_{\text {trend }}^{2}=6.20, p=0.01$ and in females, $\chi_{\text {trend }}^{2}=19.13, \mathrm{p}<0.001$. There was a suggestion that males had slightly higher rates than females after adjusting for age $(\mathrm{p}=$ $0.06)$.

H DUCREYI INCIDENCE

Of 775 people initially negative for $H$ ducreyi and with a serum sample available, 42 seroconverted. Incidence rates were 24.6 (95\% CI $11.3-28.6)$ in males and 20.0 per 1000 person years of observation (95\% CI 13.4-29.8) in females. The age adjusted rate ratio was not significant $(0.79,95 \%$ CI 0.43-1.46).

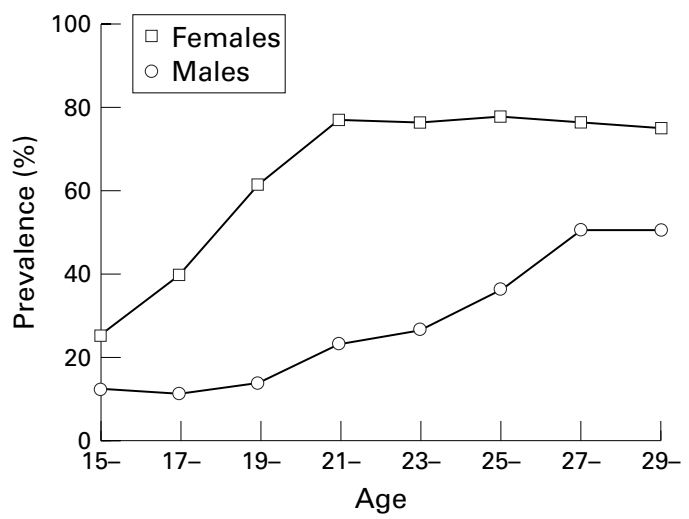

Figure $1 \mathrm{HSV}-2$ prevalence rates by age and sex (for those aged 15-30 years).

HSV-2 PREVALENCE

Results were available for 908 adults; the overall prevalence rates were $36.0 \%(31.2-41.1)$ in males and $71.5 \%$ (67.5-75.3) in females. Rates increased with age in both males and females to approximately $60 \%\left(\chi_{\text {trend }}^{2}=48.34 ; \mathrm{p}<0.001\right)$ and over $80 \%\left(\chi_{\text {trend }}^{2}=48.10 ; \mathrm{p}<0.001\right)$ respectively (table 3 ). The gradual increase with age is clearly seen in figure 1 which shows the prevalence by age and sex for those aged 15-30 years. Across all age groups the rates in males are significantly lower than in females. The peak prevalence rates are reached at about 21 years in females and much later in males, about 27 years.

HERPES SIMPLEX VIRUS TYPE 2 INCIDENCE Of 373 people initially seronegative for $\mathrm{HSV}-2$ and with sample available, 78 seroconverted, incidence rates were 73.2 (95\% CI $53.4-$ 100.3) for males and 122.9 (95\% CI 90.4$167.1)$ per 1000 person years of observation in females. The age adjusted rate ratio was 1.70 (95\% CI 1.09-2.65), $\mathrm{p}=0.02$.

ASSOCIATION BETWEEN HIV-1 SEROPOSITIVITY AND THE SEROINCIDENCE AND PREVALENCE OF OTHER STIS

Table 4 shows the association between HIV status and incidence of other STIs studied, it includes data from all the HIV incident cases studied. The rate ratio of HSV-2 incidence was 3.69 (95\% CI 2.06-6.61) in HIV seropositive cases versus HIV seronegative after adjusting for age and sex. The corresponding rate ratio for $H$ ducreyi was 3.50 (95\% CI 1.29-9.46) among female HIV positive cases versus negatives with no effect seen in males. For $T$ pallidum there were too few incident cases to make any meaningful comparison.

Table 4 Association between HIV status and incidence of other STIS

\begin{tabular}{|c|c|c|c|c|c|c|c|c|c|}
\hline \multirow[b]{3}{*}{ HIV status } & \multicolumn{9}{|c|}{ STI incidence rates/1000 pyo* } \\
\hline & \multicolumn{3}{|c|}{$T$ pallidum } & \multicolumn{3}{|l|}{$H S V-2$} & \multicolumn{3}{|c|}{ H ducreyi } \\
\hline & pyo & $i n c$ & rate & pyo & $i n c$ & rate & pyo & $i n c$ & rate \\
\hline Negative & 1760.6 & 19 & 10.8 & 813.3 & 69 & 84.8 & 1838.2 & 37 & 20.1 \\
\hline Prevalent & 77.7 & 1 & 12.9 & 15.0 & 5 & 333.3 & 70.5 & 4 & 56.7 \\
\hline Incident & 55.5 & 1 & 18.0 & 29.6 & 9 & 304.1 & 59.3 & 2 & 33.7 \\
\hline
\end{tabular}

^Person years of observation.

Rate per 1000 person years. 
Association between HIV-1 seroprevalence and prevalence of other STIs was significant (Mantel-Haenszel test) for $H$ ducreyi $(\mathrm{p}=0.01)$ and for HSV-2 $(\mathrm{p}=0.004)$ but not significant for syphilis $(\mathrm{p}>0.4)$.

HSV-2 SEROSTATUS BY AGE AND NUMBER OF LIFETIME SEXUAL PARTNERS

A total of 778 adults (289 men, 489 women) had information on reported lifetime sexual partners and HSV-2 serology available at the time of the second assessment. There was a significant association between HSV-2 seropositivity and the reported number of lifetime sexual partners after adjusting for age and sex, $\mathrm{p}=0.001$, although the effect was stronger among females than males, $p=0.003$ and $\mathrm{p}=0.08$ respectively. In females, rates of infection were high, over $60 \%$, for all numbers of reported lifetime sexual partners and increased to over $80 \%$ among those reporting two to four partners with no major increase thereafter. In males HSV-2 prevalence rose from $23 \%$ to slightly over $60 \%$ among those with $0-1$ and $10+$ lifetime sexual partners respectively.

\section{Discussion}

The results of this study have confirmed our earlier data on the descriptive epidemiology of STIs which indicated that STIs are common in this rural population. We have observed overall prevalence rates of about $13 \%$ for syphilis and about $8 \%$ for $H$ ducreyi with no differences between sexes. HSV-2 prevalence rates were very high, and in females were approximately twice those in males. These prevalence rates are against a background of stable HIV-1 prevalence of $8 \%$ in adults in the general population with an annual incidence rate of approximately $0.6 \%{ }^{17}$ The rate in the sample population studied was however lower $(4.9 \%)$, the most probable reason being that the blood from HIV positives would be more likely to have been used up in other ongoing studies. The observed finding that peak prevalence rates in females are reached by 20 years for HSV- 2 and by 25 years for syphilis, while the corresponding ages in males are 25 and 35 years respectively, indicates earlier sexual activity in females compared with males in this population.

Data on STI prevalence and particularly on incidence in general populations are generally scarce owing to difficulties associated with conducting population based longitudinal studies. This study has relied on serological testing rather than symptom recognition since we know that most STIs particularly HSV-2 do not cause easily recognisable symptoms. Our findings are based on a sample size of approximately 1000 subjects enrolled from ongoing studies of population dynamics of HIV-1 infection. Incident analysis was, however, based on slightly smaller numbers than would have otherwise been owing to a lack of sufficient volumes of samples at the different times for all individuals. The antibody assays and test algorithms were performed under rigorous quality control procedures to ensure reliable results. We are aware that the sensitivity and specificity of the $H$ ducreyi ELISA test used are not optimal. However, this test has been successfully used by several groups and has proved to be a useful tool for epidemiological studies and for definition of existing (current and past) levels of exposure. ${ }^{18}$ For $T$ pallidum and HSV-2 we used the most reliable tests available which are considered to be the gold standards. Those giving a blood sample in our surveys are more likely to be compliant than those not doing so and some were more willing to give larger samples than others. We do not think, however, that compliant people have significant differences in sexual exposure and antibody test results from those who do not. In conclusion, the results of this study, in our opinion, provide a reliable estimate of the magnitude of the STI problem in the population under study.

Most available data on STI prevalence and incidence rates are STI clinic based and there are virtually none from general populations. Similarly, systematic surveillance of STI epidemiology with the exception of HIV does not exist in sub-Saharan Africa. ${ }^{19}$ This is a handicap and makes comparison of our findings with others rather difficult. There is limited literature on syphilis data in general populations; baseline survey results from a community trial in rural Tanzania showed prevalence of active syphilis in the range of $8-9 \% .{ }^{20}$ In another community based trial of STD control for HIV-1 infection in rural south west Uganda, $10.2 \%$ of women had active or recent syphilis infection. ${ }^{21}$ Another comparable population based study, in Kagera region, Tanzania, found an overall prevalence of $5.9 \%$ for active syphilis among adults, and $13.5 \%$ for past syphilis with an overall incidence of 11.6 per 1000 person years at risk. ${ }^{22}$ In the same study a highly significant association between HIV-1 infection and syphilis was found, a finding that we did not observe in this study possibly because of smaller numbers. There is now available evidence that genital ulcerative diseases facilitate transmission and acquisition of HIV $-1^{23}{ }^{24}$ but we are not aware of any study that has looked at the association between HIV-1 status and incidence of other STIs. We have found that the risk of incidence for HSV-2 and $H$ ducreyi is much higher among HIV prevalent and incident cases than in HIV negative individuals. Similar associations have been found in other studies with the prevalence of HSV-2 shedding in HIV seropositive women being up to four times greater than in HIV seronegative women. ${ }^{25}$

The observed female-male differences for HSV-2 prevalence and incidence rates have been documented by other studies. ${ }^{26-28}$ The difference is believed to be due to the greater mucosal surface of the female genital tract, which results in higher risk of transmission to women. Males are also thought to have higher rate of disease recurrence which is likely to make them more infectious ${ }^{29}$ : the estimated risk of a susceptible female contracting HSV from an infected male is $80 \%$ following a single contact. ${ }^{30}$ It has also been suggested that there is a more vigorous immune response by women 
which may also partly explain their higher seroprevalence. ${ }^{3}$

There are difficulties in obtaining reliable population based sexual behaviour data. We have assessed the association between HSV-2 seropositivity and reported number of lifetime sexual partners to see whether this could be used as a marker of past sexual behaviour since it is a chronic and persistent infection primarily transmitted sexually. The correlation of seropositivity with number of reported lifetime sexual partners is better and significant for females, a finding that has been observed in other studies. ${ }^{31}$ In sexual behavioural intervention trials HSV-2 incidence could be used as an objective marker of sexual behaviour.

The study was funded by the Medical Research Council/ Overseas Development Administration of the United Kingdom, Global Programme on AIDS of the World Health Organisation. Contributors: Anatoli Kamali was involved in the study design, directly supervised the field data collection, and was highly involved in data analysis and writing the paper. Andrew Nunn, programme statistician, instrumental in designing the study, did programme statistician, instrumental in designing the study, dic most of statistical analysis and assisted greatly in writing th paper. Daan W Mulder, head of the Medical Research Council Programme at the time the study was conducted, was responsible for the overall direction of the study. He was the key person in the study design and gave tremendous support in writing the paper. Eddy Van Dyck was responsible for testing sera for $H$ ducreyi and $T$ pallidum in Antwerp and interpretation of tes results. In addition, he contributed to the writing of the paper. Jim G Dobbins was responsible for testing sera for HSV-2 a CDC Atlanta and interpretation of the test results. James AG Whitworth took over from Daan Mulder as the head of the proanalysis and writing the paper from the initial draft up to the time of submission.

1 Mosha F, Nicoll L, Barongo M, et al. A population-based study of syphilis and sexually transmitted disease syndromes in north-western Tanzania. 1. Prevalence and incidence. Genitourin Med 1993;69:415-20.

2 Grosskurth H, Mayaud F, Mosha F, et al. Asymptomatic gonorrhoea and chlamydial infection in rural Tanzanian men. BMF 1996;312:277-80.

3 Duncan ME, Roggen E, Tibaux G, et al. Seroepidemiological studies of Haemophilus ducreyi in Ethiopian women. cal studies of Haemophilus ducre
Sex Transm Dis 1994;21:280-8.

4 Le-Bacq F, Mason PR, Gwanzura L, et al. HIV and other sexually transmitted diseases at a rural hospital in Zimbabwe. Genitourin Med 1993;69:352-6.

5 Beherts FM, Liomba G, Lule G, et al. Sexually transmitted diseases and human immunodeficiency virus control in Malawi: a field study of genital ulcer disease. F Infect Dis 1995;171:451-5.

6 Hook EW, Cannon RO, Nahmias AJ, et al. Herpes simplex virus infection as a risk factor for human immunodeficiency virus infection in heterosexuals. 7 Infect Dis 1992;165:251-5.

7 Piot P, Van-Dyck E, Ryder RW, et al. Serum antibody to Haemophilus ducreyi as a risk factor for HIV infection in Africa, but not in Europe. International Conference on Africa, but not in Europe. International Con fer

8 Laga M, Manoka A, Kivuvu M, et al. Non-ulcerative sexually transmitted diseases as risk factors for HIV-1 transmission in women: results from a cohort study. AIDS 1993;7:95-102
9 Nzila N, Laga M, Thiam MA, et al. HIV and other sexually transmitted diseases among female prostitutes in Kinshasa. AIDS 1991;5:715-21.

10 Wagner HU, Van Dyck E, Roggen E, et al. Seroprevalence and incidence of sexually transmitted diseases in a rura Ugandan population. Int $\mathcal{F}$ STD AIDS 1994;5:332-7.

11 Nunn AJ, Mulder DW, Kamali A, et al. Mortality associated with HIV-1 infection over five years in a rural Ugandan population: cohort study. BMF 1997;315:767-71.

12 Nunn AJ, Wagner HU, Kamali A, et al. Migration and HIV-1 seroprevalence in a rural Ugandan population. AIDS 1995;9:503-6.

13 Nunn AJ, Biryahwaho B, Downing R, et al. Algorithms for detecting antibodies to HIV-1: results from a rural detecting antibodies to HIV-1: result

14 Nunn AJ, Downing R, Biryahwaho B, et al. Computerassisted quality assurance in an HIV-1 serology laboratory. Meth Inform Med 1994;33:170-3.

15 Museyi K, Van Dyck E, Vervoort T, et al. Use of an enzyme immunoassay to detect serum IgG antibodies Haemophilus ducreyi. F Infect Dis 1988;157:1039-43.

16 Sanchez-Martinez D, Schmid DS, Whittington W, et al. Evaluation of a test based on baculovirus-expressed glycoprotein $\mathrm{G}$ for detection of herpes simplex type-specific antibodies. F Infect Dis 1991;164:1196-9.

17 Kengeya-Kayondo JF, Kamali A, Nunn AJ, et al. Incidence of HIV-1 infection in adults and socio-demographic characteristics of seroconverters in a rural population in Characteristics of seroconverters in a rural population

18 Desjardins M, Thompson CE, Filion LG, et al. Standardization of an enzyme immunoassay for human antibody to Haemophilus ducreyi. f Clin Microbiol 1992;30:2019-24

19 Pham-Kanter GBT, Steinberg RC, Ballard RC. Sexually transmitted diseases in South Africa. Genitourin Med 1996; 72:160-71

20 Grosskurth H, Mosha F, Todd J, et al. A community trial of the impact of improved sexually transmitted disease treatment on the HIV epidemic in rural Tanzania: 2. Baseline survey results. AIDS 1995;9:927-34.

21 Sewankambo N, Gray RH, Wawer M, et al. HIV-1 infection associated with abnormal vaginal flora morphology and bacterial vaginosis. Lancet 1997;350:546-50.

22 Killewo JZ, Sandstrom A, Bredberg-Raden U, et al. Prevalence and incidence of syphilis and its association with HIV-1 infection in a population-based study in the With HIV-1 infection in a population-based study in the 31 .

23 Kreis J, Carael M, Meheus A. Role of sexually transmitted diseases in transmitting human immunodeficiency virus. Genitourin Med 1988;64:1-2.

24 O'Farrell N, Tovey SJ. High cumulative incidence of genita herpes amongst HIV-1 seropositive heterosexuals in south London. Int $\mathscr{f}$ STD AIDS 1995;5:415-18.

25 Augenbraun M, Feldman J, Chirgwin K, et al. Increased genital shedding of herpes simplex type 2 in HIVseropositive women. Ann Intern Med 1995;123:845-7.

26 Rawls WE, Adam E, Melnick JL. Geographical variation in the association of antibodies to herpesvirus type 2 and car-
cinoma of the cervix. In: Biggs Pm, de The G, Paynes LN, eds. Oncogenesis and herpesviruses. Scientific Publications II. eds. Oncogenesis and herpesviruses. Scientific Publications II. Lyons: In

27 Mertz GJ, Benedetti J, Ashley R, et al. Risk factors for the sexual transmission of genital herpes. Ann Intern Med 1992;116:197-202

28 Bryson Y, Dillon M, Bernstein DI, et al. Risk of acquisition of genital herpes simplex virus type 2 in sex partners of persons with genital herpes: a prospective couple study. $\mathcal{F}$ Infect Dis 1993;167:942-6.

29 Benedetti J, Corey L, Ashley R. Recurrence rates in genital herpes after symptomatic first-episode infection. Ann Intern Med 1994;121:847-54.

30 Whitley RJ, Kimberlin DW, Roizman B. Herpes simplex viruses. Clin Infect Dis 1998;26:541-55.

31 Fleming DT, McQuuillan GM, Johnson RE, et al. Herpes simplex virus type 2 in the United States, 1976 to 1994 . N Engl f Med 1997;337:1105-11. 